\title{
Lean Body Mass
}

National Cancer Institute

\section{Source}

National Cancer Institute. Lean Body Mass. NCI Thesaurus. Code C71258.

The mass of the body minus the fat. 